\title{
Spinal cavernous angioma complicated by hemorrhage: susceptibility-weighted imaging findings
}

Angioma cavernoso medular complicado por hemorragia: achados na RM ponderada em susceptibilidade magnética

Diogo Goulart Corrêa ${ }^{1}$, Emerson Leandro Gasparetto²

A 16-year-old boy presented with posterior cervical pain radiating to the right upper limb. Cervical magnetic resonance imaging (MRI) revealed an intramedullary cavernous angioma (CA) in the level of C2. Susceptibility-weighted imaging (SWI) showed longitudinal hypointensity from the bulb to the level of T1, which was suggestive of a longitudinal fiber-dissecting hemorrhage (Figure).
Intramedullary CAs are associated with an increased risk of hemorrhage. SWI is optimized for detection of magnetic susceptibility effects of blood products ${ }^{1}$. SWI is more sensitive for detection of CAs in the brain ${ }^{2}$, but the usefulness of SWI in cases of spinal CAs is not known.
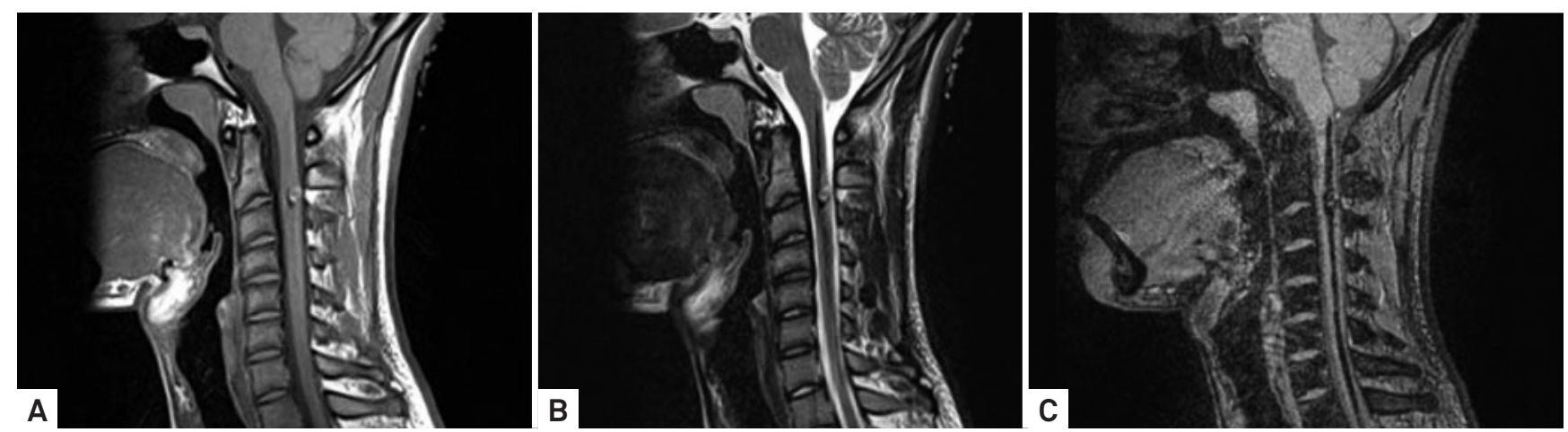

Figure. T1- (A) and T2-weighted (B) images show a nodular lesion, with a hyperintense central focus in both images and a peripheral halo of hypointensity in the T2-weighted image, suggestive of a CA. (C) SWI showing a longitudinal hypointensity in the posterior portion of the medulla, which extends from the medulla oblongata to the level of T1. The T1- and T2-weighted images do not obviously show the hemorrhage or its extension. In the T2-weighted image, the perilesional hemorrhage is characterized by a discrete longitudinal image, but SWI shows its extension more conspicuously.

References

1. Mittal S, Wu Z, Neelavalli J, Haacke EM. Susceptibility-weighted imaging: technical aspects and clinical applications, part 2. AJNR Am J Neuroradiol 2009;30:232-252.

2. de Souza JM, Domingues RC, Cruz LC Jr, Domingues FS, lasbeck T,
Gasparetto EL. Susceptibility-weighted imaging for the evaluation of patients with familial cerebral cavernous malformations: a comparison with T2-weighted fast spin-echo and gradient-echo sequences. AJNR Am J Neuroradiol 2008;29:154-158.

${ }^{1} \mathrm{MD}$, Department of Radiology, Federal University of Rio de Janeiro, Rio de Janeiro RJ, Brazil;

${ }^{2}$ MD, Ph.D, Department of Radiology, Federal University of Rio de Janeiro, Rio de Janeiro RJ, Brazil.

Correspondence: Diogo Goulart Corrêa; Rua Rodolpho Paulo Rocco 255 / Cidade Universitária / Ilha do Fundão; 21941 -913 Rio de Janeiro RJ - Brasil;

E-mail: diogogoulartcorrea@yahoo.com.br

Conflict of interest: There is no conflict of interest to declare.

Received 27 March 2013; Received in final form 26 April 2013; Accepted 03 May 2013. 